\title{
Origin of PYX thermal stability investigation with calorimetric and spectroscopic methods
}

\author{
Tomasz Gołofit $^{1}\left[\right.$ Mateusz Szala $^{2}$
}

Received: 3 April 2017/Accepted: 22 June 2017/Published online: 1 July 2017

(c) The Author(s) 2017. This article is an open access publication

\begin{abstract}
Bis(picrylamino)-3,5-dinitropyridine (PYX) is a highly thermostable explosive. The examination of its thermal decomposition was conducted, and kinetic parameters of the decomposition reaction were determined. Their values for the initial conversion degree $(\alpha=0.02)$ and hermetic conditions are ${ }_{\mathrm{a}} E_{\mathrm{a}}=261 \pm 11 \mathrm{~kJ} \mathrm{~mol}^{-1}$, $\log A=18.09$. Volatile semi-products are formed during PYX thermal decomposition, which influence kinetic parameters of the decomposition reaction. That is why hermetic pans should be used for this analysis. The maximum safe temperature, i.e., $T=246{ }^{\circ} \mathrm{C}$, was calculated on the basis of ADT24 method. Analysis of results obtained during spectroscopic analysis of PYX indicates that there are three main reasons of the compound stability. Strong hydrogen bonds between oxygen atoms in nitro groups and aromatic $\mathrm{C}-\mathrm{H}$ and $\mathrm{N}-\mathrm{H}$ protons are confirmed by analysis of IR and ${ }^{1} \mathrm{H}$ NMR spectra. Large couplings in PYX carbon skeleton which stabilize the molecule are detected on the basis of FWHM of ${ }^{13} \mathrm{C}$ NMR peaks. The stability of the analyzed compound is enhanced by the lack of carbon with $s p^{3}$ hybridization.
\end{abstract}

Keywords. Thermal decomposition · SADT - ADT24 · Kinetic parameters . Thermal explosion

Tomasz Gołofit

tomgol@ch.pw.edu.pl

1 Faculty of Chemistry, Warsaw University of Technology, Noakowskiego 3, 00-664 Warsaw, Poland

2 Faculty of New Technologies and Chemistry, Military University of Technology, Kaliskiego 2, 00-908 Warsaw, Poland

\section{Introduction}

Highly energetic materials (HEM) are employed in the civil, cosmic, as well as military technology to carry out explosions. HEM should be insensitive to external stimuli in order for them to be used safely. High thermal stability is demanded in numerous applications, e.g., oil and natural gas exploration or in cosmic technology. 2,6-Bis(picrylamino)-3,5-dinitropyridine (PYX) is an example of a compound which meets these requirements [1]. Its structure is presented in Fig. 1.

Despite the fact that PYX was first obtained in 1970s, there is still little data about this compound [2]. Information about thermal properties of PYX is particularly significant, because this compound is a commonly acknowledged potential component of thermostable mixtures. HEM processing, storage and application pose a threat of thermal explosion. This phenomenon occurs as the result of the accumulation of heat released during the decomposition reaction [3]. To estimate the hazard related to this process, kinetic parameters of the decomposition reaction should be determined. The Kissinger method is one of the easiest and currently employed methods of kinetic parameters determination [4]. This method assumes the single value of kinetic parameters for the entire decomposition process. Such information for PYX is available [5]. Many parallel and subsequent reactions run during HEM decomposition and that is why the values of kinetic parameters may substantially change with the conversion degree of the progress [6-8]. Thermal explosion may occur even in a low conversion degree [9], so the determination of kinetic parameters at the initial stage of decomposition is significant. For this reason, the kinetic parameters determined by the Kissinger method should not be used to estimate the risk of a thermal explosion. In 1995, 
<smiles>O=[N+]([O-])c1cc([N+](=O)[O-])c(Nc2nc(Nc3c([N+](=O)[O-])cc([N+](=O)[O-])cc3[N+](=O)[O-])c([N+](=O)[O-])cc2[N+](=O)[O-])c([N+](=O)[O-])c1</smiles>

Fig. 1 Structure of PYX molecule

the kinetic parameters of the PYX decomposition reaction for the solid phase $\left(E=224.5 \mathrm{~kJ} \mathrm{~mol}^{-1}, \log A=15.3\right)$ were determined based on the Russian manometric method [10]. The kinetic parameters obtained from such measurements describe the kinetics of the release of gaseous decomposition products. The threat of thermal explosion is related to exothermic reactions and not to the evolution of gaseous decomposition products. Therefore, to estimate the risk of uncontrolled exothermic decomposition it is more appropriate to use kinetic parameters derived from DSC measurements rather than manometric ones. Data obtained from DSC measurements allow the determination of the maximum safe operating temperature of TMS and adiabatic decomposition temperature for a 24-h induction time ADT24 [11-14], self-accelerating decomposition temperature (SADT) [13-20]. SADT is the lowest ambient temperature at which the stored substance in a particular commercial packaging will self-heat and the temperature increase will be at least $6 \mathrm{~K}$ in 7 days or less. SADT is therefore related to the heat output generated in the system and the heat output to the environment. Transport packages may contain from several dozen to several hundred kilograms of a given substance. Experiments with such a large amount of substances are difficult and dangerous to carry out, so a test in a $500 \mathrm{~cm}^{3}$ Dewar vessel was performed. Such vessels are characterized by heat losses comparable to that of a transport packaging containing approximately $100 \mathrm{~kg}$ of a given substance [20]. SADT can also be determined using a smaller sample mass based on the adiabatic storage test and the isothermal storage test. Another method of estimating SADT is DSC [15-19] measurements. ADT24 and TMS temperatures describe the risk of uncontrolled adiabatic decomposition. Adiabatic conditions are the most unfavorable conditions in terms of the risk of thermal explosions.

There is little information in the literature on the initial thermal decomposition of PYX. In order to estimate the safety of use and storage, as well as the risk of thermal explosion of PYX, the determination of the kinetic parameters of the initial decomposition stage and the compound's heat capacity is required.
The aim of this work was to investigate the process of PYX thermal decomposition under different conditions, determine of kinetic parameters of the decomposition reaction, as well as to estimate the application and storage safety of PYX. Obtaining knowledge about the microscopic origins of PYX thermal stability through spectroscopic measurements of PYX molecules and their interactions was the second goal of these studies.

\section{Experimental}

\section{Materials}

PYX was synthesized in the Division of High Energetic Materials at the Warsaw University of Technology. The purity of compound was determined with HPLC and was equal to ca. $99 \%$.

\section{Instrumental analysis}

Differential scanning calorimetry (DSC) analyses were carried out with the TA Instruments Q2000 calorimeter. Measurements were taken with airtight aluminum pans with a nitrogen flow of $50 \mathrm{~mL} \mathrm{~min}{ }^{-1}$. Thermogravimetric analyses (TG) were carried out with a TA Instruments SDT Q600 device. These measurements were taken in aluminum pans with a nitrogen flow of $100 \mathrm{~mL} \mathrm{~min}{ }^{-1}$. DSC and TG studies were performed in five different rates of the temperature increase in the range of $\beta=0.5-8{ }^{\circ} \mathrm{C} \mathrm{min}^{-1}$ in the following range of temperatures: $200-500{ }^{\circ} \mathrm{C}$. Measurements with extreme rates of the temperature increase were repeated threefold, according to the recommendations of the Conference of Thermal Analysis and Calorimetry (ICTAC) [7].

\section{Kinetic parameters calculation methods}

Kinetic parameters of the decomposition reaction of analyzed systems were determined using Thermokinetics 3.1 NETZSCH program. The results indicate that the kinetic parameters are dependent on all of the subsequent and parallel reactions. Therefore, the obtained value of the activation energy is henceforth referred to as the apparent activation energy $\left({ }_{\mathrm{a}} E_{\mathrm{a}}\right)[7,8,21]$. In accordance with to ICTAC recommendations [7], the apparent activation energy ${ }_{\mathrm{a}} E_{\mathrm{a}}$ was determined with the Friedman isoconversion method [22]. A first-order reaction model was assumed in the calculation of the preexponential factor.

Thermokinetics 3.1 NETZSCH software also allows one to determine kinetic parameters using the Kissinger method. In the Kissinger method, a single value of kinetic 
parameters for the whole process is obtained. Because the thermal explosion hazard relates to the initial decomposition stage, this method was not used in the calculation.

\section{Maximum safe temperature of technological processes performance}

ADT24 is the temperature, at which a given system undergoes thermal explosion after $24 \mathrm{~h}[11,12]$ under adiabatic conditions. The following equation describing the time to explosion $\left(t_{\mathrm{ex}}\right)$ parameter may be derived on the basis of this theory:

$t_{\mathrm{ex}}=\left(\frac{c_{\mathrm{p}} R T^{2}}{\Delta H_{\mathrm{D}} A E_{\mathrm{a}}}\right) \exp \left(\frac{E_{\mathrm{a}}}{R T}\right)$

where $E_{\mathrm{a}}$-activation energy, $A$-preexponential factor of Arrhenius equation, $T$-material temperature, $R$-universal gas constant, $\Delta H_{\mathrm{D}}$-reaction heat, $c_{\mathrm{p}}$-sample heat capacity. Temperature $20 \mathrm{~K}$ lower than value determined with ADT24 method is acknowledged to be the maximum safe temperature of technological processes performance.

\section{Self-accelerating decomposition temperature}

Equations that allow the determination of SADT are available in the literature [15]. In the first step, the temperature of no return $\left(T_{\mathrm{NR}}\right)$ is determined according to Eq. 2. Then SADT is calculated according to Eq. 3.

$$
\begin{aligned}
& \frac{R \cdot T_{\mathrm{NR}}^{2}}{\left(\frac{\mathrm{d} T}{\mathrm{~d} t}\right)_{T_{0}} \cdot E_{\mathrm{a}}}=\frac{R \cdot T_{\mathrm{NR}}^{2} \cdot C_{\mathrm{p}}}{\Delta H_{\mathrm{r}} \cdot A \cdot \exp \left(-E_{\mathrm{a}} / R \cdot T_{\mathrm{NR}}\right) \cdot f(\alpha) \cdot E_{\mathrm{a}}} \\
& =\frac{m \cdot C_{\mathrm{p}}}{U \cdot S} \\
& \mathrm{SADT}=T_{\mathrm{NR}}-\frac{R \cdot T_{\mathrm{NR}}^{2}}{E_{\mathrm{a}}}
\end{aligned}
$$

For a standard transport package containing $50 \mathrm{~kg}$ of substance $(m)$, a heat transfer coefficient $(U)$ of $5 \mathrm{~W} \mathrm{~m}^{-2} \mathrm{~K}^{-1}$ and a heat transfer surface $(S)$ of $1.4 \mathrm{~m}^{2}$ were assumed.

\section{Nuclear magnetic resonance}

NMR experiments were conducted in a Bruker AvanceIII HD $500 \mathrm{MHz}$ spectrometer (field $11.7 \mathrm{~T}$ ). ${ }^{1} \mathrm{H}$ and ${ }^{13} \mathrm{C}$ spectra were referenced to tetramethylsilane (TMS, $\delta$ $0.00 \mathrm{ppm}$ ) protons and carbons, respectively. ${ }^{15} \mathrm{~N}$ chemical shifts are referenced to liquid ammonia. Spectra of PYX dissolved in DMSO- $\mathrm{d}_{6}$ were measured at room temperature with sweeps $15 \mathrm{ppm}\left({ }^{1} \mathrm{H}\right), 300 \mathrm{ppm}\left({ }^{13} \mathrm{C}\right)$ and $500 \mathrm{ppm}$ $\left({ }^{15} \mathrm{~N}\right)$; the number of scans depends on nuclei sensitivity (64-8192). Spectra were acquired and processed using standard Bruker software (TopSpin 3.1.).

\section{Infrared spectroscopy}

The Fourier transform infrared spectroscopy (FTIR) analysis was carried out using Thermo Scientific NICOLET 6700 spectrometer. The disk for analysis was prepared by triturating and pressing $1 \mathrm{mg}$ of PYX sample and $200 \mathrm{mg}$ potassium bromide. The spectrum was recorded in the wave number range $4000-400 \mathrm{~cm}^{-1}$.

\section{Results and discussion}

\section{Calorimetric studies}

TG-DSC studies of PYX were performed in an open pan. The first run was carried out for the sample with $2 \mathrm{mg}$ mass with the temperature increase rate $8{ }^{\circ} \mathrm{C} \min ^{-1}$. Uncontrolled decomposition was noted, which indicates high apparent activation energy and high thermal effect of PYX decomposition. Sample mass was gradually decreased. However, even for $0.4 \mathrm{mg}$ the decomposition still runs uncontrollably. That is why measurements performed at the temperature increase rate in the range of $\beta=0.5$ to $\beta=4{ }^{\circ} \mathrm{C} \min ^{-1}$ were applied to determine kinetic parameters. Figure 2 presents the typical TG-DSC curve obtained for the temperature increase rate of $\beta=4{ }^{\circ} \mathrm{C} \mathrm{min}{ }^{-1}$.

The mass loss process is two-step and begins at the temperature of $340{ }^{\circ} \mathrm{C}$. The extrapolated start of the mass loss equals $T_{\text {onsetTG }}=366.6 \pm 0.4{ }^{\circ} \mathrm{C}$, whereas the maximum of the mass loss rate takes place at $T_{\text {peakTG }}=$ $371.2 \pm 0.1^{\circ} \mathrm{C}$. This process is exothermal, $T_{\text {onsetDSC }}=$ $366.7 \pm 0.4{ }^{\circ} \mathrm{C}, T_{\text {peakDSC }}=371.9 \pm 0.2{ }^{\circ} \mathrm{C}$, transformation enthalpy $\Delta H=0.98 \pm 0.05 \mathrm{~kJ} \mathrm{~g}^{-1}$. A series of TGDSC runs was performed for different temperature increase rates. The mass loss was related to the exothermal effect for

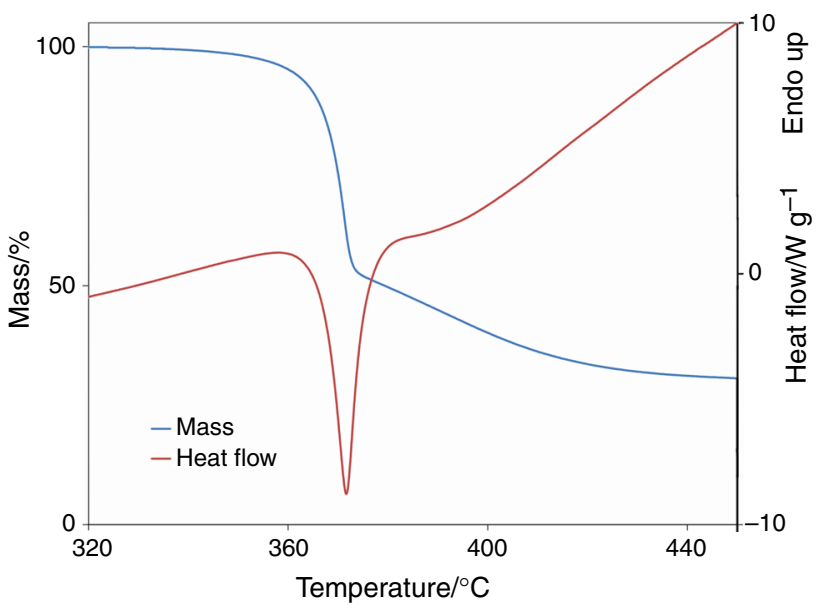

Fig. 2 Typical TG and DSC curves of the PYX sample obtained for the temperature increase rate of $\beta=4{ }^{\circ} \mathrm{C} \mathrm{min}{ }^{-1}$ 


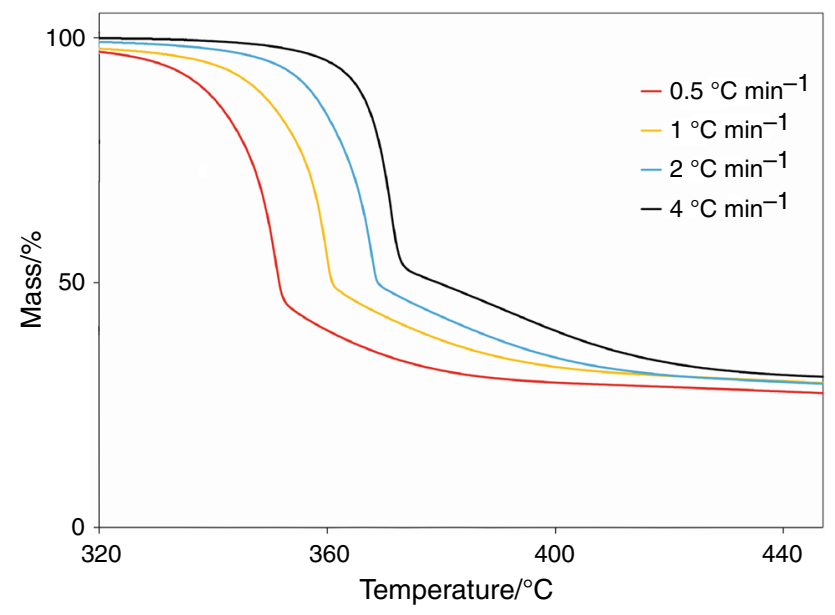

Fig. 3 TG curves of PYX samples obtained for the temperature increase rates $\beta=0.5,1,2,4{ }^{\circ} \mathrm{C} \min ^{-1}$, measurements in open pans

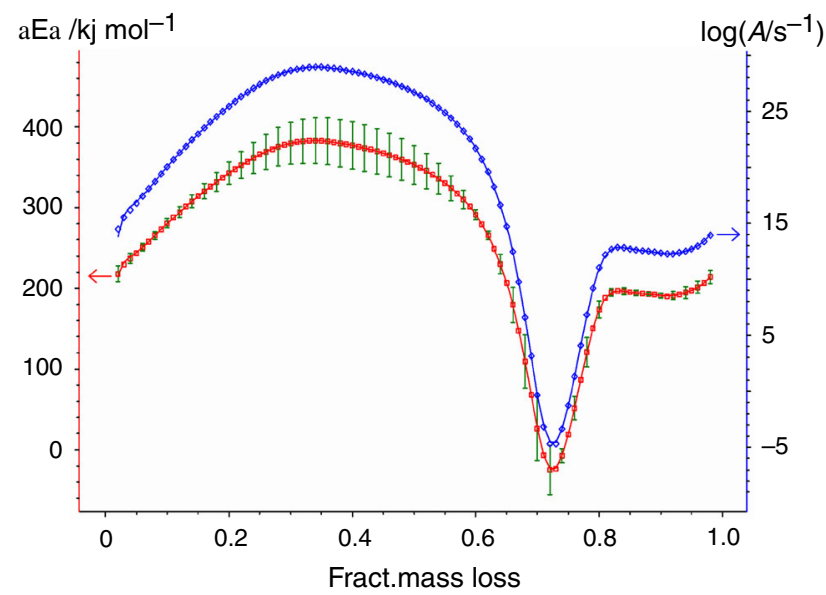

Fig. 4 Dependence of the apparent activation energy $\left({ }_{\mathrm{a}} E_{\mathrm{a}}\right)$ and preexponential factor $\log (\log A)$ on the conversion degree $(\alpha)$ of PYX sample on the basis of TG measurements in the open pan

all of the measurements. The obtained TG curves are presented in Fig. 3.

As expected, the mass loss process shifts in the direction of lower temperatures with the rate decrease. A two-step decomposition process was observed for all measurements. A black, flocculent substance was the residue of the sample after each run. Its mass at the temperature of $550{ }^{\circ} \mathrm{C}$ was dependent on the temperature increase rate and equalled $19-25 \%$ of the initial sample mass. Kinetic parameters of running the process were determined on the basis of obtained TG curves. Changes of apparent activation energy and the preexponential factor depending on the conversion degree are shown in Fig. 4.

The apparent activation energy at the initial stage $(\alpha=0.02)$ equals ${ }_{\mathrm{a}} E_{\mathrm{a}}=218 \pm 10 \mathrm{~kJ} \mathrm{~mol}^{-1}$ and gradually increases with an increase of the conversion degree reaching ${ }_{\mathrm{a}} E_{\mathrm{a}}=383 \pm 29 \mathrm{~kJ} \mathrm{~mol}^{-1}$ for $\alpha=0.34$. Then it decreases

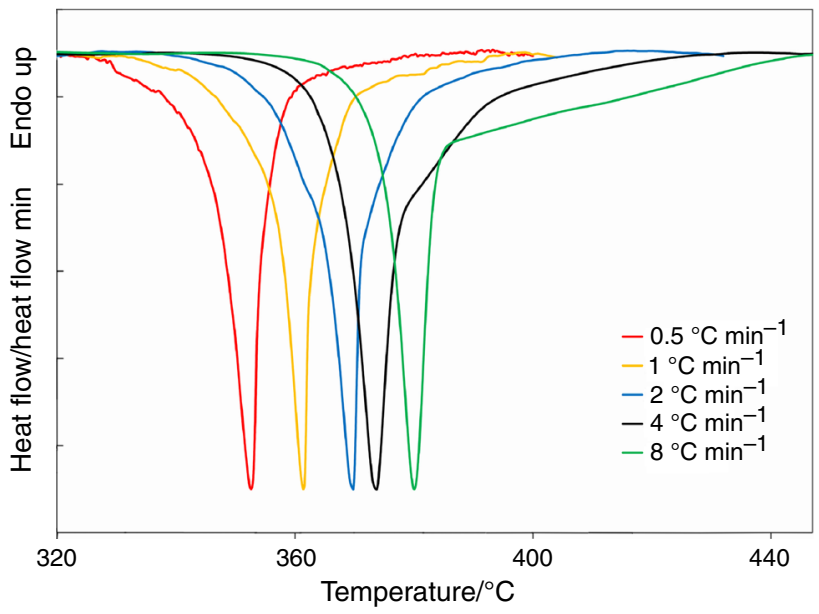

Fig. 5 DSC curves of PYX decomposition obtained at the temperature increase rates $\beta=0.5,1,2,4,8{ }^{\circ} \mathrm{C}$ min $^{-1}$, measurements in hermetic pans

and rises again. Minimum value of the apparent activation energy is ${ }_{\mathrm{a}} E_{\mathrm{a}}=-25 \pm 31 \mathrm{~kJ} \mathrm{~mol}^{-1}$ for $\alpha=0.72$. The change in $\log \mathrm{A}$ exhibits a similar trend as the change in the apparent activation energy, for $\alpha=0.02 \log A=14.48$, for $\alpha=0.34 \log A=28.95$, for $\alpha=0.72 \log A=-4.69$. Such significant changes of the apparent activation energy with increasing conversion degree are related to the bulk of reactions running during PYX thermal decomposition. The obtained values of the kinetic parameters determined in TG measurements may also be influenced by the high vapor pressure of intermediate decomposition products or PYX. The influence of measurement conditions on the determination of kinetic parameters has already been described in the literature [8]. The determined kinetic parameters for the initial PYX thermal decomposition step are in line with those obtained using the Russian manometric method [10].

Series of the DSC measurements of PYX thermal decomposition in hermetic pans was performed at different temperature increase rates. The obtained DSC curves are presented in Fig. 5.

Two-step exothermal decomposition process of PYX samples was observed for all measurements. Parameters calculated at the temperature increase rate of $4{ }^{\circ} \mathrm{C} \mathrm{min}{ }^{-1}$ were as follows: $T_{\text {onsetDSC }}=367.1^{\circ} \mathrm{C} T_{\text {peakDSC }}=373.7^{\circ} \mathrm{C}$, transformation enthalpy $\Delta H=2.85 \mathrm{~kJ} \mathrm{~g}^{-1}$. PYX decomposition temperatures measured in hermetic and open pans are consistent (Fig. 2). Almost threefold higher decomposition thermal effect was noted in the case of measurement performed in the hermetic pan. It is the evidence of high vapor pressure of PYX or its intermediate decomposition products. Kinetic parameters of the decomposition reaction were determined on the basis of the obtained DSC curves. Dependence of the apparent activation energy and preexponential factor on the conversion degree is shown in Fig. 6. 


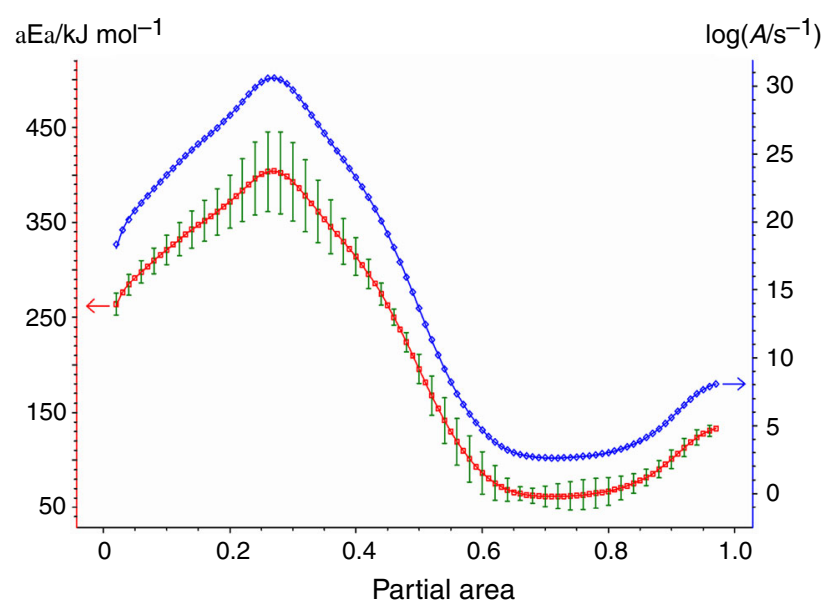

Fig. 6 Dependence of the apparent activation energy $\left({ }_{\mathrm{a}} E_{\mathrm{a}}\right)$ and preexponential factor $\log (\log A)$ on the conversion degree $(\alpha)$ of PYX sample on the basis of DSC measurements in a hermetic pan

At the initial stage $(\alpha=0.02)$ the apparent activation energy is ${ }_{\mathrm{a}} E_{\mathrm{a}}=261 \pm 11 \mathrm{~kJ} \mathrm{~mol}^{-1}$ and gradually increases with the increase of the conversion degree reaching ${ }_{\mathrm{a}} E_{\mathrm{a}}=392 \pm 38 \mathrm{~kJ} \mathrm{~mol}^{-1}$ for $\alpha=0.27$. Then it decreases to ${ }_{\mathrm{a}} E_{\mathrm{a}}=58 \pm 9 \mathrm{~kJ} \mathrm{~mol}^{-1}$ for $\alpha=0.69$. A similar character of changes was observed for the preexponential factor $\log$. It equals 18.09 for $\alpha=0.02$. Significant changes of the apparent activation energy with the conversion degree result from many different reactions during PYX thermal decomposition. Discrepancies in the course of these changes for different pan types (hermetic and open) observed for $\alpha>0.3$ confirm the influence of high vapor pressure of intermediate products on the course of decomposition process. Values of the apparent activation energy on the basis of studies made in hermetic pans are much higher in relation to results obtained for samples in open pans.

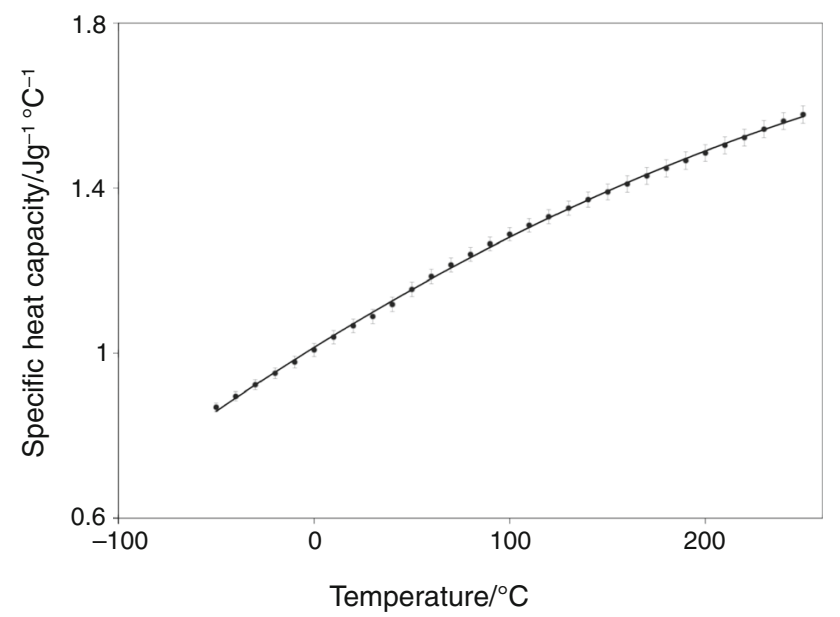

Fig. 7 Specific heat capacity of PYX as the function of temperature
Heat capacity is a significant parameter necessary to estimate the hazard related to the thermal explosion of unstable compounds. That is why PYX specific heat capacity was determined. Figure 7 shows the mean value obtained from three measurements.

Specific heat capacity of PYX in the range of temperatures from -50 to $250{ }^{\circ} \mathrm{C}$ may be described with the following equation: $c_{\mathrm{p}}(T)_{\mathrm{PYX}}=1.014+2.944 \times 10^{-3} \mathrm{~T}$ $+2.826 \times 10^{-6} \mathrm{~T}^{2}$, with the correlation coefficient of 0.9994. Determined kinetic parameters and specific heat capacity of PYX allow the estimation of the maximum safe temperature of technological processes with PYX participation, according to Eq. (1). The calculated value is $T=246{ }^{\circ} \mathrm{C}$. The SADT of PYX was calculated based on Eq. 3 for a transport tank containing $50 \mathrm{~kg}$ of this substance. It equals $276{ }^{\circ} \mathrm{C}$. As expected, the designated SADT value is higher than TMS. The obtained temperatures are very high which confirms the high thermal stability of PYX.

\section{Nuclear magnetic resonance study}

Figures 8-10 show proton, carbon and nitrogen NMR spectra of pure PYX. The ${ }^{1} \mathrm{H}$ NMR spectrum of PYX is shown in Fig. 8.

Three diagnostic peaks are observed with chemical shifts: 8.92 ; 9.21 and $11.26 \mathrm{ppm}$. The ratio of the peak areas of the signals is practically equal $4: 1: 1$. The first and second peak can be assigned to aromatic $\mathrm{C}-\mathrm{H}$ protons in picryl groups and to the single proton in the pyridine ring. The broad signal with small height and maximum at $11.26 \mathrm{ppm}$ can be assigned to two $\mathrm{N}-\mathrm{H}$ protons in the picrylamino system. Broadening of the signal and peak area smaller than theoretical indicates that those amino protons undergo chemical exchange and can dissociate. Similar chemical shifts of $\mathrm{N}-\mathrm{H}$ protons are observed in

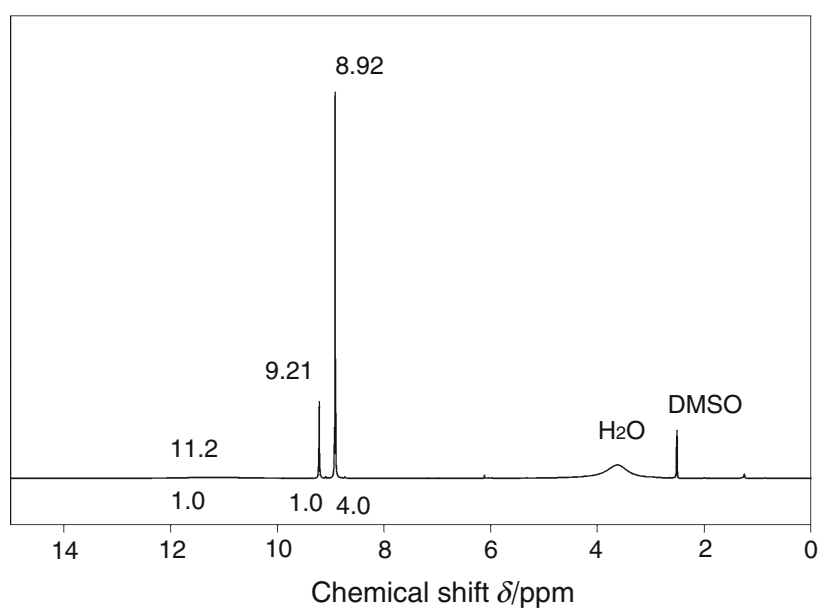

Fig. $8{ }^{1} \mathrm{H}$ NMR spectrum of PYX in DMSO-d 6 


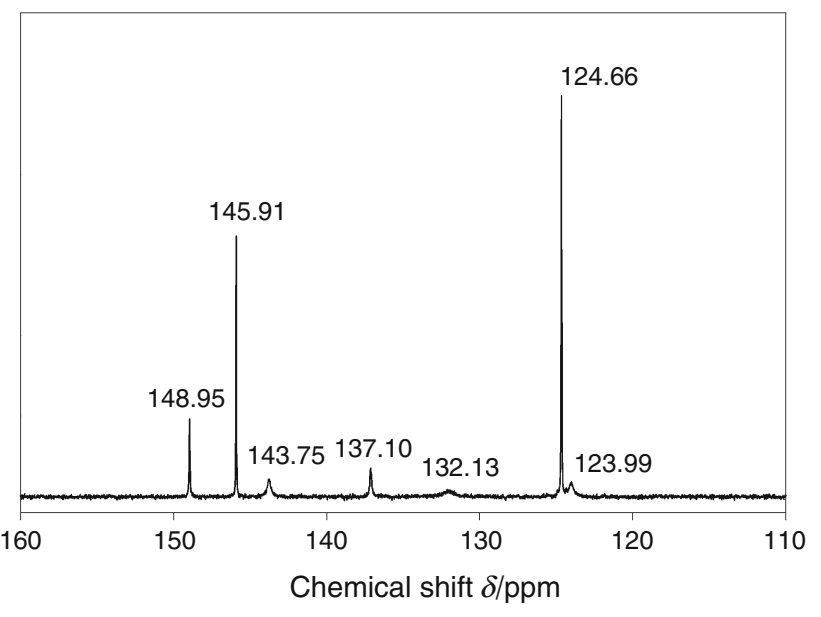

Fig. $9{ }^{13} \mathrm{C}$ NMR spectrum of PYX in DMSO- $\mathrm{d}_{6}$

3-nitro-1,2,4-triazol-5-one [23] and the measured pKa of its compounds is 3.98 [24]. Dissociation ability of PYX N-H protons was confirmed by synthesis of its organic and inorganic salt [1].

Chemical shift of aromatic protons in PYX picryl substituents $(8.92 \mathrm{ppm})$ is smaller in relation to appropriate protons in relative compounds like 2,4,6-trinitroaniline (9.06 ppm), $N$-metyl- $N, 2,4,6$-tetranitroaniline $(9.33 \mathrm{ppm})$, 1,3-diamino-2,4,6-trinitrobenzene $(9.12 \mathrm{ppm})$. A reverse tendency is observed for the single proton in pyridine. The chemical shift of the proton in position 4 of the pyridine ring $(9.21 \mathrm{ppm})$ is higher than in analogs like 2,6-diamino3,5-dinitropyridine (9.00 ppm) [25], 3,5-dinitropyridine
(9.13 ppm) [26]. Two different shielding tendencies indicate that between PYX molecules there are strong interactions and most importantly hydrogen bonds are present even in diluted solutions. The full investigation of intraand intermolecular hydrogen bonds between PYX molecules requires a separate extensive study.

The ${ }^{13} \mathrm{C}$ NMR spectrum of PYX is shown in Fig. 9. Seven signals with different width and intensity are observed. A small broad peak at $123.99 \mathrm{ppm}$ represents carbons connected to the nitro groups in position 2,6 in picryl substituents. A sharp peak at $124.66 \mathrm{ppm}$ is typical for carbons connected with nitro group in position 3,5 of a trinitrophenyl group, whose intensity is enhanced by the NOE effect (Silverstein). The next small and broad peak at $132.13 \mathrm{ppm}$ can be assigned to carbon in position 2 of the pyridine ring. The signal at $137.12 \mathrm{ppm}$ represents the single $\mathrm{C}-\mathrm{H}$ carbon in pyridine ring. Carbons which are located next to nitrogen in the pyridine ring give a resonance signal at $143.75 \mathrm{ppm}$. The next sharp and intensive peak at $145.91 \mathrm{ppm}$ can be assigned to carbons connected to nitrogen in position 1 of the picryl rings. The most deshielded signal, i.e., the one at $148.95 \mathrm{ppm}$, can be assigned to carbons in position 4 of the picryl substituent. General analysis of carbon NMR signals intensities of PYX indicated that carbons nuclei in pyridine have longer relaxation times than appropriate carbon nuclei in other pyridine derivatives. This phenomenon indicates that in PYX molecules there is substantial coupling between pyridine and the benzene ring. This may be responsible for chemical and thermal stability of PYX.
Fig. $10{ }^{15} \mathrm{~N}$ NMR spectrum of PYX in DMSO-d 6

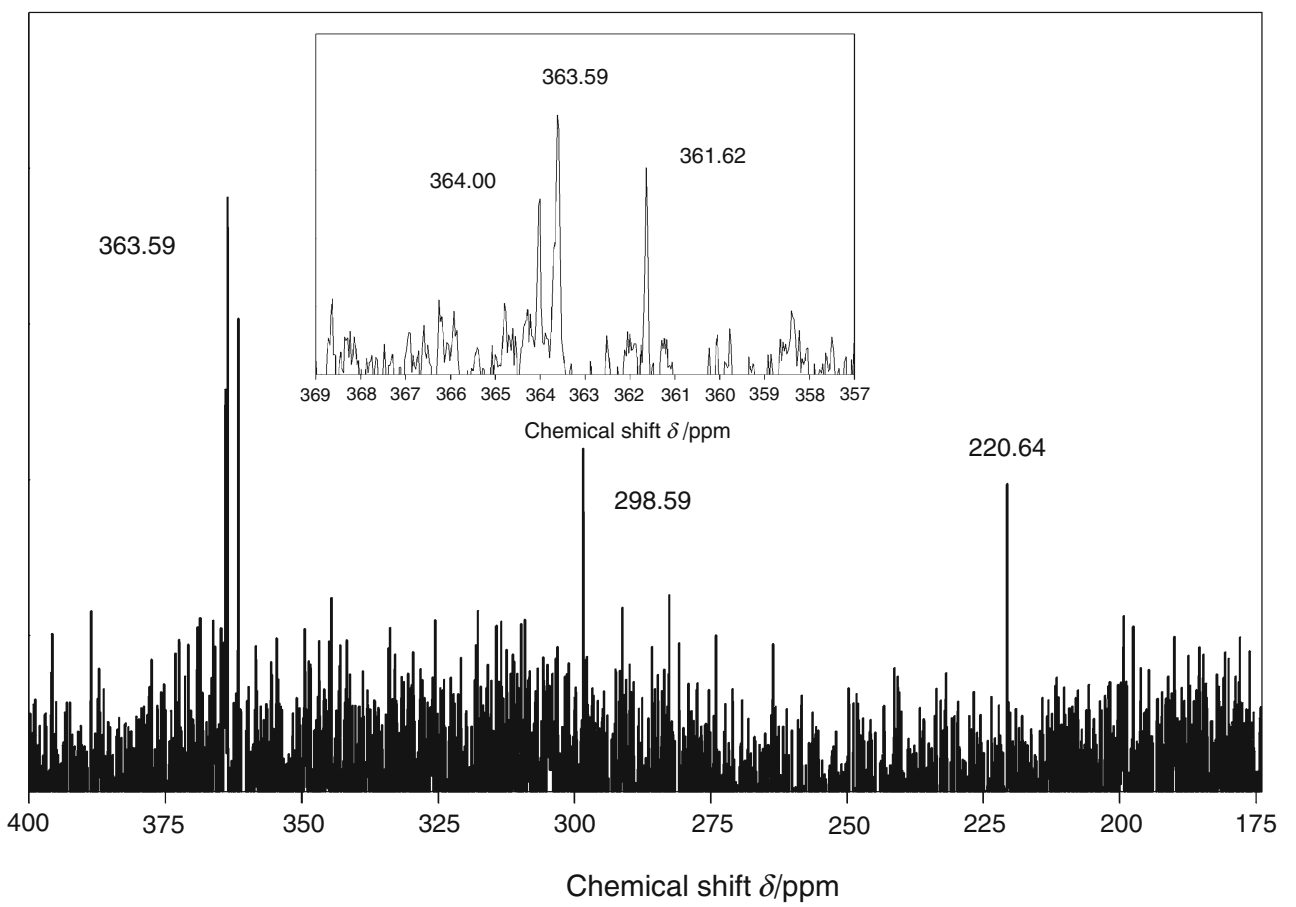


Table 1 Characteristic IR bands identified on PYX spectrum

\begin{tabular}{|c|c|c|}
\hline Wavenumber $/ \mathrm{cm}^{-1}$ & Relative intensity & Interpretation \\
\hline 3270 & Medium & $v_{\text {str }} \mathrm{N}-\mathrm{H}$ \\
\hline 3084 & Medium & $v_{\text {str }} \mathrm{C}-\mathrm{H}$ \\
\hline 1635 & Strong & $v_{\text {def }} \mathrm{N}-\mathrm{H}$ \\
\hline 1594 & Strong & $v_{\text {as }} \mathrm{C}=\mathrm{N}$ \\
\hline 1548 & Strong & $v_{\text {as }} \mathrm{NO}_{2}$ \\
\hline 1539 & Strong & $v_{\text {as }} \mathrm{NO}_{2}$ \\
\hline 1491 & Strong & $v_{\text {str }} \mathrm{C}=\mathrm{C}$ \\
\hline 1481 & Strong & $v_{\text {str }} \mathrm{C}=\mathrm{N}$ \\
\hline 1432 & Medium & $v_{\text {str }} \mathrm{C}=\mathrm{C}$ \\
\hline 1401 & Strong & $v_{\mathrm{s}} \mathrm{NO}_{2}$ \\
\hline 1346 & Strong & $v_{\mathrm{s}} \mathrm{NO}_{2}$ \\
\hline 1332 & Strong & $v_{\mathrm{s}} \mathrm{NO}_{2}$ \\
\hline 1309 & Strong & $v_{\text {str }} \mathrm{C}_{\mathrm{Ar}}-\mathrm{N}$ \\
\hline 1292 & Medium & $v_{\text {str }} \mathrm{C}_{\mathrm{Ar}}-\mathrm{N}$ \\
\hline 1243 & Weak & $v_{\text {def }} \mathrm{C}_{\mathrm{Ar}}-\mathrm{H}$ \\
\hline 1193 & Weak & $v_{\text {def }} \mathrm{C}_{\mathrm{Ar}}-\mathrm{H}$ \\
\hline 1092 & Weak & $v_{\text {def }} \mathrm{C}_{\mathrm{Ar}}-\mathrm{H}$ \\
\hline 949 & Weak & $v_{\text {def }} \mathrm{C}_{\mathrm{Ar}}-\mathrm{H}$ \\
\hline 922 & Weak & $v_{\text {com }} \mathrm{C}_{\mathrm{Ar}}-\mathrm{C}_{\mathrm{Ar}}$ \\
\hline 910 & Weak & $v_{\text {com }} \mathrm{C}_{\mathrm{Ar}}-\mathrm{C}_{\mathrm{Ar}}$ \\
\hline 858 & Weak & $v_{\mathrm{str}} \mathrm{C}_{\mathrm{Ar}}-\mathrm{N}\left(\mathrm{NO}_{2}\right)$ \\
\hline 837 & Weak & $v_{\text {def }} \mathrm{C}_{\mathrm{Ar}}-\mathrm{H}$ \\
\hline 827 & Weak & $v_{\text {def }} \mathrm{C}_{\mathrm{Ar}}-\mathrm{H}$ \\
\hline 780 & Weak & $v_{\text {def }} \mathrm{C}_{\mathrm{Ar}}-\mathrm{H}$ \\
\hline 761 & Medium & $v_{\text {def }} \mathrm{C}_{\mathrm{Ar}}-\mathrm{H}$ \\
\hline 720 & Medium & $v_{\text {def }} \mathrm{C}_{\mathrm{Ar}}-\mathrm{H}$ \\
\hline 697 & Weak & $v_{\text {def }} \mathrm{C}_{\mathrm{Ar}}-\mathrm{H}$ \\
\hline 679 & Weak & $v_{\text {def }} \mathrm{C}_{\mathrm{Ar}}-\mathrm{H}$ \\
\hline
\end{tabular}

The ${ }^{15} \mathrm{~N}$ NMR spectrum of PYX is shown in Fig. 10. Nitrogen nuclei located in $\mathrm{N}-\mathrm{H}$ bridges are represents by the signal at $220.64 \mathrm{ppm}$. Analogical nitrogen nuclei in $2,2^{\prime}, 4,4^{\prime}, 6,6^{\prime}$-hexanitrodiphenylamine give a resonance signal at $214.2 \mathrm{ppm}$, which means that the same nitrogen nuclei are deshielded in the PYX molecule. There are two reasons of this phenomenon, the first is the steric factor and the second are hydrogen bonds between amino proton and oxygens in nitro groups. The nitrogen located in the pyridine ring can be assigned to resonance $298.59 \mathrm{ppm}$. Signal with a chemical shift of $361.63 \mathrm{ppm}$ represents nitrogen nuclei of nitro groups in positions 4 of the picryl groups. The signal at $363.59 \mathrm{ppm}$ can be assigned to four nitro groups in positions 2,6 of the picryl substituents. The last signal, i.e., the one at $364.02 \mathrm{ppm}$, represents 2 nitro groups in the pyridine ring. The structure of a PYX molecule caused strong deshielding of nitrogen nuclei in pyridine nitro groups. The chemical shifts of nitrogen nuclei in PYX nitro groups are typical for aromatic nitrocompounds.

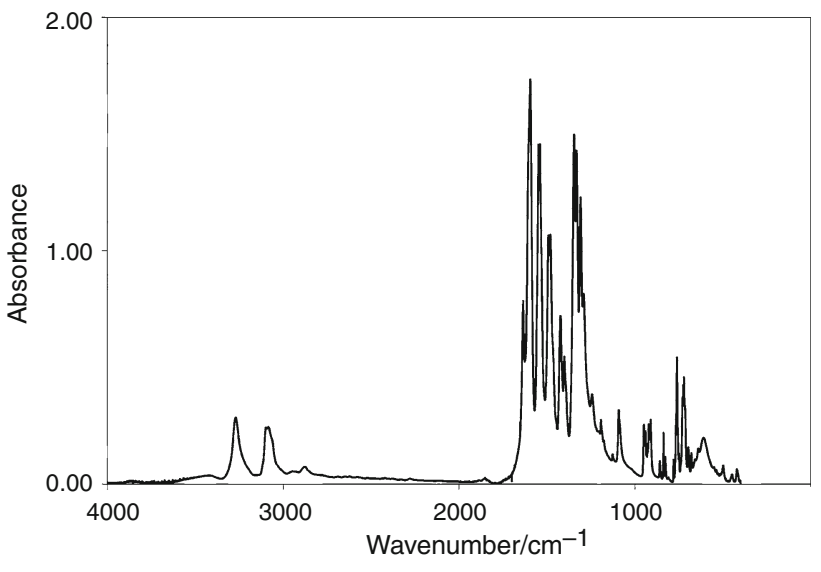

Fig. 11 Solid phase infrared (FTIR) spectrum of PYX

\section{Infrared spectroscopy}

The characteristic vibrations bands and their interpretation were collected in Table 1. In accordance with the triple ring PYX molecule structure, the IR spectrum is complicated (Fig. 11).

Many types of vibrations have their characteristic bands in two narrow spectral regions, i.e., the diagnostic region: $1700-1300 \mathrm{~cm}^{-1}$ and the fingerprint region. The presence of strong hydrogen bonds caused a shift of the appropriate bands to lower frequencies (i.e., $v_{\text {as }} \mathrm{NO}_{2}$ ) with changes in their intensities. The other characteristic bands which represent functional groups in PYX give bands with typical wavenumbers. General conclusion which can be drawn after IR spectrum analysis is that the origin of PYX stability is inter- and intramolecular hydrogen bonds and lack of carbon with $s p^{3}$ hybridization.

\section{Conclusions}

PYX is a highly thermostable compound. Its decomposition runs in the temperature range of $340-380{ }^{\circ} \mathrm{C}$, depending on the conditions. Kinetic parameters of reactions running during PYX thermal decomposition were determined. Their values for the initial conversion degree $(\alpha=0.02)$ and hermetic conditions are equal to ${ }_{\mathrm{a}} E_{\mathrm{a}}=261 \pm 11 \mathrm{~kJ} \mathrm{~mol}^{-1}, \log A=18.09$. Numerous parallel and subsequent reactions occur during PYX thermal decomposition, which causes substantial changes in the determined kinetic parameters. It is therefore important to use the kinetic parameters determined for the initial decomposition stage to estimate PYX safety. Volatile semiproducts are formed as the result of PYX thermal decomposition. They influence the kinetic parameters of determined kinetic parameters of the decomposition reaction when untight pans are applied. For the initial degree of 
conversion $(\alpha=0.02)$ and unhermetic conditions, they are ${ }_{\mathrm{a}} E_{\mathrm{a}}=218 \pm 10 \mathrm{~kJ} \mathrm{~mol}^{-1}$ and $\log A=14.48$, respectively. The maximum safe temperature of technological process performance was calculated on the basis of the ADT24 method and determined parameters, and its value was $T=246{ }^{\circ} \mathrm{C}$. The self-accelerating decomposition temperature of PYX, as determined for a transport tank containing $50 \mathrm{~kg}$ of the substance, is equal to $276^{\circ} \mathrm{C}$. It is very likely that the process may run in an uncontrolled manner because of high activation energy of the decomposition reaction during PYX thermal analysis, so caution must be maintained.

Analysis of results obtained during spectroscopic analysis of PYX indicates that there are three main reasons of the compound stability. Strong hydrogen bonds between oxygen atoms in nitro groups and aromatic $\mathrm{C}-\mathrm{H}$ and $\mathrm{N}-\mathrm{H}$ protons are confirmed by analysis of IR and ${ }^{1} \mathrm{H}$ NMR spectra. Moreover, substantial coupling in PYX carbon skeleton, which stabilizes the molecule, is detected on the basis of FWHM of ${ }^{13} \mathrm{C}$ NMR peaks. Stability of the analyzed compound is further enhanced by lack of carbon with $s p^{3}$ hybridization.

Open Access This article is distributed under the terms of the Creative Commons Attribution 4.0 International License (http://crea tivecommons.org/licenses/by/4.0/), which permits unrestricted use, distribution, and reproduction in any medium, provided you give appropriate credit to the original author(s) and the source, provide a link to the Creative Commons license, and indicate if changes were made.

\section{References}

1. Klapötke TM, Stierstorfer J, Weyrauther M, Witkowski TG. Synthesis and investigation of 2,6-bis (picrylamino)-3,-dinitropyridine (PYX) and its salts. Chem Eur J. 2016;22(25):8619-26.

2. Coburn MD, Singleton JL. Picrylamino-substituted heterocycles. V. Pyridines. J Heterocycl Chem. 1972;9(5):1039-44.

3. Semenov NN. Chemical kinetics and chain reactions. London: Oxford University Press; 1935.

4. Kissinger HE. Reaction kinetics in differential thermal analysis. Anal Chem. 1957;29(11):1702-6.

5. Keshavarz MH, Zohari N, Seyedsadjadi SA. Validation of improved simple method for prediction of activation energy of the thermal decomposition of energetic compounds. J Therm Anal Calorim. 2013;114(2):497-510.

6. Gołofit T, Zyśk K. Thermal decomposition properties and compatibility of CL-20 with binders HTPB, PBAN, GAP and polyNIMMO. J Therm Anal Calorim. 2015;119:1931-9.

7. Vyazovkin S, Burnham AK, Criado JM, Pérez-Maqueda LA, Popescu C, Sbirrazzuoli N. ICTAC Kinetics Committee recommendations for performing kinetic computations on thermal analysis data. Thermochim Acta. 2011;520:1-19.

8. Gańczyk K, Zygmunt A, Gołofit T. Thermal properties of TEX decomposition or sublimation. $\mathrm{J}$ Therm Anal Calorim. 2016;125:967-75.
9. Abramov VG, Vaganova NI. Effect of a side reaction with small heat liberation on the critical thermal-explosion condition of the main selfcatalyzed reaction. Fiz Goreniya Vzryva. 1978;14(5):135-41.

10. Falyakhov IF, Sharnin GP, Safin NM, Saifullin ISh, Fassakhov $\mathrm{RKh}$. Investigation of thermal stability of high-melting thermostable explosives. In: Proceedings of the XXVth international pyrotechnics seminar, pp. 187-201. Moscow; 1995 (in Russian).

11. Grewer T, Klusacek H, Löffler U, Rogers RL, Steinbach J. Determination and assessment of the characteristic values for the evaluation of the thermal safety of chemical processes. J Loss Prev Process Ind. 1989;2(4):215-23.

12. Germain A, Bizzarri D, Dodet C. Thermal decomposition of ethyl and methyl parathion. J Loss Prev Process Ind. 2000;13(1):1-5.

13. Lakshminarasimhan T. Predicting 24 and $8 \mathrm{~h}$ adiabatic decomposition temperature for low temperature reactions by kinetic fitting of nonisothermal heat data from reaction calorimeter (RC1e). Org Process Res Dev. 2014;18(2):315-20.

14. Kikuchi T. The use of the ARC to estimate safe handling and transportation temperatures. J Jpn Soc Saf Eng. 2001;40:100-7 (in Japanese).

15. Lu GB, Yang T, Chen LP, Zhou YS, Chen WH. Thermal decomposition kinetics of 2-ethylhexyl nitrate under nonisothermal and isothermal conditions. J Therm Anal Calorim. 2016;124(1):471-8.

16. Zhang GY, Liu JC, Song NM, Liu Y, Yang L. Research on the thermal performance and storage life of series of high-energy hydrazine nitrate complexes. J Therm Anal Calorim. 2017. doi:10.1007/s10973-017-6323-3.

17. Pourmortazavi SM, Farhadi K, Mirzajani V, Mirzajani S, Kohsari I. Study on the catalytic effect of diaminoglyoxime on thermal behaviors, non-isothermal reaction kinetics and burning rate of homogeneous double-base propellant. J Therm Anal Calorim. 2016;125(1):121-8.

18. Abusaidi H, Ghaieni HR, Pourmortazavi SM, Motamed-Shariati $\mathrm{SH}$. Effect of nitro content on thermal stability and decomposition kinetics of nitro-HTPB. J Therm Anal Calorim. 2016;124(2):935-41.

19. Pourmortazavi SM, Rahimi-Nasrabadi M, Rai H, Jabbarzadeh Y, Javidan A. Effect of nanomaterials on thermal stability of 1,3,6,8tetranitro carbazole. Cent Eur J Energ Mater. 2017;14(1):201-16.

20. Grewer T. Thermal hazards of chemical reactions, industrial safety series. Amsterdam: Elsevier; 1994.

21. Xiao LB, Zhao FQ, Luo Y, Gao HX, Li N, Meng ZH, et al. Thermal behavior and safety of 4,10-dinitro-2,6,8,12-tetraoxa4,10-diazaisowutrzitane. J Therm Anal Calorim. 2015;121:839-42.

22. Friedman HL. Kinetics of thermal degradation of char-forming plastics from thermogravimetry. Application to a phenolic plastic. J Polym Sci Polym Symp. 1964;6(1):183-95.

23. Szala M, Szymańczyk L. Analysis of common explosives in different solvents by nuclear magnetic resonance spectroscopy. Cent Eur J Energ Mater. 2014;11(1):129-42.

24. Zbarsky VL, Yudin NV. Kinetics of the synthesis of NTO in nitric acid. Propellants Explos Pyrotech. 2005;30(4):298-302.

25. Wang Y, Hu Z, Meng X, Jing J, Song Y, Zhang C, Huang Y. A simple and efficient route for preparing 2,3,5,6-tetraaminopyridine hydrochloride salt. Molecules. 2009;14(5):1652-9.

26. Sturala J, Bohacova S, Chudoba J, Metelkova R, Cibulka R. Electron-deficient heteroarenium salts: an organocatalytic tool for activation of hydrogen peroxide in oxidations. J Org Chem. 2015;80(5):2676-99. 